\title{
COMMENTARY
}

\section{Pulmonary Veno-Occlusive Disease}

\section{A Rare Cause of Pulmonary Hypertension}

\author{
Kazuyuki Yahagi and Renu Virmani
}

From the CVPath Institute, Gaithersburg, Maryland

Pulmonary veno-occlusive disease (PVOD) is a rare cause of pulmonary hypertension $(\mathrm{PH})$, characterized by increased pulmonary artery pressure contributing to right heart failure and death. ${ }^{1}$ Historically, PVOD has been described by diverse terms, such as isolated pulmonary venous sclerosis, obstructive disease of the pulmonary veins, or venous form of primary pulmonary hypertension (PPH). The true incidence of PVOD is uncertain, considering that a large number of PPH cases are misclassified. The estimated frequency of PVOD is between 5\% to 25\%; however, $10 \%$ incidence of PVOD in a pooled analysis of seven studies totaling 465 PPH cases examined between 1970 and 1991 has also been reported. ${ }^{2}$ The noninvasive diagnosis of PVOD in cases confirmed by histology-identified clinical features of pulmonary arterial hypertension $(\mathrm{PAH})$ include: $>10$ pack per year smoking history, high-resolution CT showing nodular ground glass opacities, septal lines, lymph node enlargement, and pleural effusion with occult alveolar hemorrhage confirmed by bronchoalveolar lavage. ${ }^{3}$

In 1975, the World Health Organization (WHO) further classified pulmonary hypertension $(\mathrm{PH})$ into primary or secondary PH. In 1998, the WHO established a clinical classification of $\mathrm{PH}$ to include five groups, which share similar pathological and hemodynamic etiologies and therapeutic approaches to treatment. ${ }^{4}$ The clinical classification of PH includes i) PAH, ii) PH due to left heart disease, iii) PH due to lung disease and/or hypoxia, iv) chronic thromboembolic pulmonary hypertension, and v) $\mathrm{PH}$ with unclear multifactorial mechanism. This consensus has remained the same with some modifications published in 2009 and during the 2013 meeting of the Fifth World Symposium. ${ }^{1}$

Major categories of PAH include sporadic or idiopathic $\mathrm{PAH}$, heritable PAH, and PAH attributed to drugs and toxins, congenital heart disease, and others. The PAH classification also includes PVOD classified as Group 1, which is characterized by structural narrowing or occlusion of the pulmonary veins and the lobular septa accompanied by loose and edematous tissue initially, which matures into dense and collagen-rich fibrous tissue. ${ }^{2}$ Considering the incidence of PVOD is $5 \%$ to $10 \%$, this estimate yields an annual percent incidence of 0.1 to 0.2 cases per million in the general population. ${ }^{5}$ Currently, multiple underlying causes of PVOD have been implicated and include infections, genetic factors, toxic exposures, thrombotic diathesis, and autoimmune disorders. ${ }^{2}$ The epidemiology, etiology, pathology, and clinical feature of PVOD, however, are not fully understood.

\section{PVOD in Humans}

In this issue of The American Journal of Pathology, Ranchoux et $\mathrm{al}^{6}$ reviewed the French PH network of all documented cases of PVOD to determine the most likely chemotherapeutic agent involved in the development of PVOD. They report that of the 179 eligible articles on PVOD, only $27(15 \%)$ could be considered chemotherapy-induced. From the French PH network, another 10 cases of POVD were added, and therefore 37 cases formed the basis for determining the most frequent chemotherapeutic agent that

\footnotetext{
Supported by CVPath Institute, Inc. (Gaithersburg, MD).

Accepted for publication November 25, 2014.

Disclosures: R.V. receives research support from Abbott Vascular, BioSensors International, Biotronik, Boston Scientific, Medtronic, MicroPort Medical, OrbusNeich Medical, SINO Medical Technology, and Terumo Corporation; has speaking engagements with Merck; receives honoraria from Abbott Vascular, Boston Scientific, Lutonix, Medtronic, and Terumo Corporation; and is a consultant for 480 Biomedical, Abbott Vascular, Medtronic, and W.L. Gore. K.Y. reports no conflicts of interest relevant to the topic of this manuscript.

Address correspondence to Renu Virmani, M.D., CVPath Institute, Inc., 19 Firstfield Rd., Gaithersburg, MD 20878. E-mail: rvirmani@cvpath.org.
} 
induced PVOD. Candidate chemotherapeutic agents attributed to POVD included alkylating agents, antimetabolites, plant alkaloid and naturally occurring molecules, and cytotoxic antibiotic and related molecules.

Of the 37 cases of chemotherapy-associated PVOD, 84\% involved alkylating or alkylating-like agents. Nearly half (43\%) were represented by cyclophosphamide (CP), followed by near equal frequency of mitomycin (24.3\%) and cisplatin $(21.6 \%)$ thus implicating $\mathrm{CP}$ as the most frequent contributing underlying chemotherapeutic agent for the development of PVOD. Chemotherapy-induced PVOD was more frequent in younger patients (4 to 66 years old; 13 individuals above 50 years; median age 37.8 years) independent of sex (male, $45.9 \%$, versus female, 54.1\%). Moreover, approximately $78 \%$ of chemotherapy-induced PVOD in the French PH network presented within 1 year following the initiation of chemotherapy.

Review of autopsy or surgical biopsy material from the French $\mathrm{PH}$ network showed muscularization and intimal thickening in septal vein, hypertrophy of the media, intimal fibrosis of pulmonary arteries located adjacent to the bronchioles, and concentric muscularization of microvessels. Furthermore, there was accumulation of hemosiderin-laden intraalveolar macrophages. This comprehensive analysis prompted the authors to further explore the possibility of developing an animal model to better understand the mechanisms involved in the induction of CP-induced PVOD.

\section{Current Animal Models of PH}

Previous animal models of PPH using monocrotaline have been criticized for not leading to successful therapies because the changes in the pulmonary arteries did not translate to those seen in humans. ${ }^{7,8}$ However, a recently described model of surgically induced PH in the rat via a shunt from the left common carotid to left jugular vein produced changes of medial hypertrophy and intimal proliferation, which may lead to improved therapy because adequate reagents will be more likely to be available for testing.

Ranchoux et $\mathrm{al}^{6}$ administered $\mathrm{CP}$ in three different animal models (mouse, rat, and rabbit), in which they reproduced morphological characteristics of PVOD similar to humans. They used these three models due to recognized morphological differences of pulmonary veins between rodents and rabbits whereby rodents have muscularized pulmonary veins whereas rabbits have thin fibrous vein walls that more closely resemble human pulmonary veins.

\section{Mouse Model}

Ranchoux et $\mathrm{al}^{6}$ initially tested the possibility of inducing PVOD by i.p. injection of single dose of $350 \mathrm{mg} / \mathrm{kg} \mathrm{CP}$ in mice with follow-up studies after 4 weeks of injection. The study showed significant increase in right ventricular systolic pressure, accompanied by compensatory right ventricular hypertrophy (RVH). Histologic findings in the lung consisted of septal thickening with accumulation of foamy intraalveolar macrophages.

\section{Rat Model}

Male and female rats received a single injection of $350 \mathrm{mg} / \mathrm{kg}$ $\mathrm{CP}$ by i.p. injection and were studied after 4 weeks, as in the mouse model above. The female rats developed marked $\mathrm{RVH}$, whereas the males had heterogeneous RVH. Moreover, a dose-response relationship to CP was seen in female rats that were dosed for two weeks with escalating concentrations of 100, 150, 200, and $250 \mathrm{mg} / \mathrm{kg}$ per week. Findings showed there was $100 \%$ mortality at the highest dose accompanied by significantly lower cardiac output, higher total pulmonary vascular resistances, and significantly higher incidence of distal microvessels occlusion confirmed by histology. In addition, serum vascular endothelial growth factor levels, soluble E-selectin, and von Willebrand factor were significantly increased in the highest dose group although other serum parameters (soluble intercellular adhesion molecule 1, monocyte chemotactic protein-1, troponin-T and -I, tissue inhibitor of metalloproteinase-1, plasminogen activator inhibitor-1, myeloperoxidase, IL-6, and tumor necrosis factor $\alpha$ ) failed to show significant change. Lung tissues from animals receiving the two highest doses also demonstrated greater 5-hydroxytryptamine levels.

Clinical heritable PAH may be related to missense mutation in $K C N K 3$ (the gene encoding potassium channel subfamily $\mathrm{K}$, number 3 ) with a resulting loss of function. The authors further corroborated that rats exposed to $\mathrm{CP}$ also had a decreased expression of $\mathrm{KCNK} 3$ protein in the lung ${ }^{6}$ and therefore must be congratulated for successfully establishing a sound model of PVOD in the rat. However, since the morphology of pulmonary veins in the rat does not resemble that of humans, they further verified their findings in a rabbit model, which has similar pulmonary vein morphology to humans.

\section{Rabbit Model}

Similar to the rat model, female rabbits that survived for 8 weeks following exposure to $100 \mathrm{mg} / \mathrm{kg} \mathrm{CP}$ doses at 0,1 , and 3 weeks showed medial hypertrophy of muscular pulmonary artery, neomuscularization of distal microvessels, and congestion and hyperplasia of septa, accompanied by significant thickening and adventitial fibrosis of pulmonary veins with vasculitis together with moderate $\mathrm{PH}$ and $\mathrm{RVH}$.

\section{Limitations and Advantages of Current Animal Models}

Although animal models of $\mathrm{PH}$ have been in existence for a long time, many investigators argue their validity, as there is a limited understanding of $\mathrm{PH}$ in humans. Despite 
criticisms, some animal models have led to clinically relevant treatments. There is no doubt that animal models of $\mathrm{PH}$ also have contributed to understanding of the pathophysiology of hypertension. ${ }^{9}$ A good example is mice that develop severe $\mathrm{PH}$ and $\mathrm{RVH}$ as a result of expressing a dominantnegative type 2 allele for bone morphogenetic protein receptor on pulmonary artery smooth muscle cells. Resultant lung changes show muscularization of small pulmonary arteries including plexiform lesions with elevated Rho/Rhokinase activity. Targeted treatment with Rho-kinase inhibitor Fasudil (Asahi Kasei Pharma, Tokyo, Japan) at $100 \mathrm{mg} /$ $\mathrm{kg}$ per day for 14 days alleviates $\mathrm{PH}$. The article by Ranchoux et $\mathrm{al}^{6}$ illustrates a similar example where $\mathrm{CP}$-induced PVOD in rats was ameliorated by simultaneously administering amifostine, a cytoprotective adjuvant used in cancer chemotherapy and radiotherapy.

\section{Potential for Prevention of PVOD Using Cytoprotective Agents}

The clinical onset of CP-induced PVOD occurs rapidly within the first year of treatment concomitant with other chemotherapeutic agents or immunosuppressants. However, it is concerning that the current available treatments for PVOD are unsatisfactory. Pulmonary vasodilators, immunosuppressive medications, anticoagulants, and oxygen therapy do not halt the disease; lung transplantation is the only recourse to prolonging the life of patients with PVOD, but with limited experience. ${ }^{10}$

Ranchoux et $\mathrm{al}^{6}$ administered cytoprotective agent mesna (an organosulfur compound used as an adjuvant in cancer chemotherapy to detoxify metabolities of $\mathrm{CP}$ and amifosline) in combination with chemotherapy to reduce normal tissue toxicity for the prevention of PVOD. The percentage of occluded distal microvessels and inflammation (CD45 intensity) were significantly less for amifostine and mesna as compared with no treatment. However, amifostine but not mesna ameliorated CP-induced $\mathrm{PH}$ with a significant improvement in survival and pulmonary hemodynamics (increase in cardiac output with decrease in total pulmonary resistances) in rats. Furthermore, only amifostine decreased the pathological pulmonary accumulation of 5-hydroxytryptamine in CP-exposed animals.

The study by Ranchoux et $\mathrm{al}^{6}$ underscores the careful assessment of patients receiving multiple chemotherapeutic drugs to determine commonalities that may be involved in the induction of a new disease, which can be detrimental for survival. Moreover, the authors developed animal models based on registries of patients presenting with PVOD with prior treatment involving chemotherapeutic agents. We believe this research is just beginning to unravel potential molecular markers that could be responsible for PVOD. Also, the generations of animal models reminiscent of human disease are badly needed if we are to make inroads into treatment of rarities, like PVOD. Further development of effective therapies is almost exclusively dependent on translational animal models, which closely mimic human disease [ie, not only morphologically, but also show common mechanism(s)]. Furthermore, we need confirmation that similar therapies shown to ameliorate the disease in the rat model are also efficacious in humans.

\section{References}

1. Simonneau G, Gatzoulis MA, Adatia I, Celermajer D, Denton C, Ghofrani A, Gomez Sanchez MA, Krishna Kumar R, Landzberg M, Machado RF, Olschewski H, Robbins IM, Souza R: Updated clinical classification of pulmonary hypertension. J Am Coll Cardiol 2013, 62: D34-D41

2. Mandel J, Mark EJ, Hales CA: Pulmonary veno-occlusive disease. Am J Respir Crit Care Med 2000, 162:1964-1973

3. Montani D, Achouh L, Dorfmuller P, Le Pavec J, Sztrymf B, Tchérakian C, Rabiller A, Haque R, Sitbon O, Jaïs X, Dartevelle P, Maître S, Capron F, Musset D, Simonneau G, Humbert M: Pulmonary veno-occlusive disease: clinical, functional, radiologic, and hemodynamic characteristics and outcome of 24 cases confirmed by histology. Medicine(Baltimore) 2008, 87:220-233

4. Simonneau G, Galie N, Rubin LJ, Langleben D, Seeger W, Domenighetti G, Gibbs S, Lebrec D, Speich R, Beghetti M, Rich S, Fishman A: Clinical classification of pulmonary hypertension. J Am Coll Cardiol 2004, 43:5S-12S

5. Rubin LJ: Primary pulmonary hypertension. N Engl J Med 1997, 336 $111-117$

6. Ranchoux B, Gunther S: Chemotherapy-induced pulmonary hypertension: role of alkylating agents. Am J Pathol 2015, 185: $356-371$

7. Liu C, Yan Z, Fang C, Cao G, Wang B, Li S, Wu S: Establishment and comparison of two reliable hyperkinetic pulmonary hypertension models in rabbits. J Thorac Cardiovasc Surg 2014

8. Wang Y, Jing L, Zhao XM, Han JJ, Xia ZL, Qin SC, Wu YP, Sun XJ Protective effects of hydrogen-rich saline on monocrotaline-induced pulmonary hypertension in a rat model. Respir Res 2011, 12:26

9. Maarman G, Lecour S, Butrous G, Thienemann F, Sliwa K: A comprehensive review: the evolution of animal models in pulmonary hypertension research; are we there yet? Pulm Circ 2013, 3: 739-756

10. Stewart S, McNeil K, Nashef SA, Wells FC, Higenbottam TW, Wallwork J: Audit of referral and explant diagnoses in lung transplantation: a pathologic study of lungs removed for parenchymal disease. J Heart Lung Transplant 1995, 14:1173-1186 